\title{
DIAGNOSTIC VALUE OF ROUTINE BONE SCINTIGRAPHY RENAL IMAGING IN RENAL CELL CARCINOMA
}

\author{
MICIIAEL B. CHANCELLOR, M.D. \\ JOHN W. KONNAK, M.D. \\ H. BARTON GROSSMAN, M.D.
}

From the University of Michigan Medical Center, Department of Surgery, Section of Urology, Ann Arbor, Michigan

\begin{abstract}
Technetium-99m-phosphate compounds used in bone scanning are excreted by the kidney, and excellent renal images can be obtained on routine bone scintigrams. The preoperative bone scans of 49 patients who underwent radical nephrectomy for renal cell carcinoma between 1981 and 1985 were reviewed for renal imaging. Ninety-four percent of the patients had abnormal bone scan renal images ( $82 \%$ had focal decreased uptake, and $12 \%$ had focal increased uptake). Six percent of the renal images were symmetrical bilaterally. When bone scans are employed in the postoperative follow-up of patients with renal cancer, they can be used to assess the status of the remaining kidney.
\end{abstract}

Bone scans have proved to be more sensitive in the detection of bone metastases than conventional $x$-ray studies. ${ }^{1}$ For this reason bone scans are used routinely in the diagnostic workup of renal cell carcinoma. The technetium-99mphosphate compounds used for bone scanning are excreted by the kidneys and often yield excellent renal images. Unsuspected renal abnormalities can be detected on renal images obtained during a routine bone scan. ${ }^{2}$ Several authors have reported results of renal images obtained incidentally during routine bone scanning. ${ }^{3-7}$ These studies were nonselective retrospective reviews of bone scans over a period of time, and only a few cases of renal cell carcinomas, presenting as a focal decreased uptake on the renal images, were reported in each study. We reviewed preoperative bone scans in 49 patients with known renal cell carcinomas. The review findings are reported.

\section{Material and Methods}

From 1981 to 1985,49 patients at the University of Michigan Medical Center had radical nephrectomy for renal cell carcinomas. All pa- tients had preoperative routine bone scans which were reviewed. The bone scans were obtained three to four hours after intravenous administration of $10-15 \mathrm{mCi}$ of ${ }^{99 \mathrm{~m}} \mathrm{Tc}$-stannousphosphate compounds (polyphosphate, pyophosphate, and diphosphate). The images were obtained with a rectilinear scanner.

The bones scans were reviewed independently of the radiographs and correlated retrospectively. Renal uptake of ${ }^{99 m}$-Tc-phosphate compounds were considered to be normal when it was symmetrical, homogenous, and sufficient to allow adequate visualization of both kidneys. Abnormal images were categorized as focal decreased uptake or focal increased uptake, then correlated with radiographs and surgical specimens. The size of the tumor images on the bone scans were also measured and compared with the actual tumor size obtained from the surgical specimens.

\section{Results}

The mean age of the 49 patients was sixtyone years, with a range from thirty-five to eighty-one. There were 32 men and 17 women. 

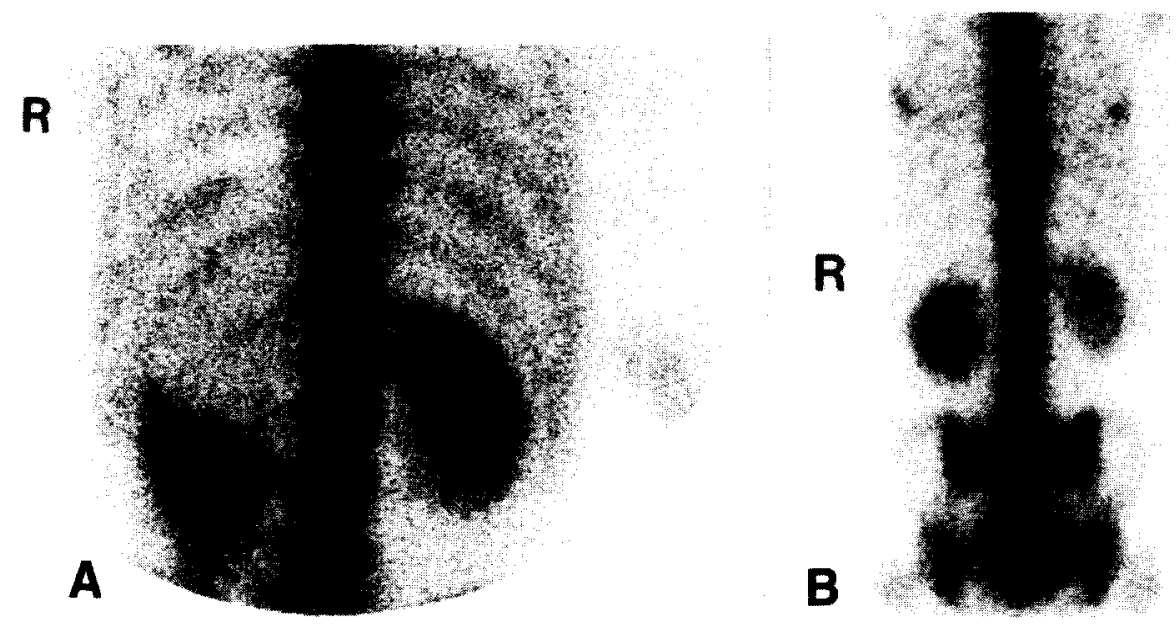

Figure 1. (A) ${ }^{99 m} T$ c bone scan of thirty-five-year-old woman with right upper pole hypernephroma showing decreased uplake and caliceal distortion. (B) Sixty-one-yearold woman with large hypernephroma involving upper two thirds of right kidney with perinephric and renal vein extension of tumor. No hydronephrosis noted on IVP or ultrasound. Bone scan showed increased uptake of right kidney, especially the upper two thirds of kidney.

\section{Comment}

The incidental discovery of renal abnormalities on routine bone scans has been described in the literature. ${ }^{1-8}$ Demonstration of absent renal activity, small kidneys, displaced kidneys, focal decrease in renal activity (mass lesions), and hydronephrosis are reliably detected on bone scans. ${ }^{6}$ When renal images are considered to be normal on routine bone scan, the level of confidence is 98 percent that the kidneys are normal. When the renal images show some abnormalities, the level of confidence is 84 percent that the kidneys are abnormal. ${ }^{5}$

In our patients with renal cell carcinomas we noted 94 percent abnormal renal images on preoperative bone scans. The mean actual tumor size was $7 \mathrm{~cm}$ and $8 \mathrm{~cm}$ in the decreased uptake and increased uptake group, respectively, as compared with only $4 \mathrm{~cm}$ in the symmetrical group. Because bone scan images are usually $1 / 6-1 / 10$ of the actual body size, the evaluation of small changes in parenchymal density are more difficult. The finding of three symmetrical normal scans in our study may be due to the small size of these tumors.

It is unclear why 12 percent of the patients with abnormal scans had increased intensity instead of the decreased activity seen in the majority of the scans. The two groups were similar in mean tumor size, pathologic histology, staging, and vascularity and the absence of obstruction by the tumor. It is important for clinicians to be aware that renal tumors can present either as increased or decreased activity on bone scan renal imaging.

While we do not advocate the use of bone scans in the workup of renal masses, our data indicate that renal tumors can be detected on routine bone scans in 94 percent of cases, a 
number comparable with excretory urography or renal ultrasound. ${ }^{9}$ Renal abnormalities should be looked for when bone scans are employed for other reasons and appropriate studies instituted if they are found. Another use may be in the follow-up of the remaining kidney in patients with renal cell carcinoma treated surgically. If bone scanning is employed in the follow-up of these patients in an effort to detect bony metastases, the renal images obtained may provide adequate follow-up of the remaining kidney, eliminating the need for excretory urography or other methods of renal imaging. Comparison with preoperative bone scan renal images should provide sensitivity similar to that seen in this study.

1500 E. Medical Center Drive Ann Arbor, Michigan 48109-0330

(DR. KONNAK)

\section{References}

1. Cole AT, Mandell J, Fried FA, and Stabb FV: The place of bone scan in the diagnosis of renal cell carcinoma, J Urol 114: 364 (1975)

2. Ilattner RS, Miller SW, and Schimmel D: Significance of renal asymmetry in bone scans: experience in 795 cases, $\mathrm{J}$ Nucl Med 15: 161 (1975).

3. Park CH, Glassman LM, and Thompson NL: Reliability of renal imaging obtained incidentally in ${ }^{99 m} \mathrm{Tc}$-polyphosphate bone scanning. J Nucl Med I4: 534 (1973).

4. Maher FA: Evaluation of renal and urinary tract abnormalities noted on scintiscans, Mayo Clin Proc 50: 370 (1975).

5. Vieras F, and Boyd CM: Diagnostic value of renal imaging incidental to bone scintigraphy with ${ }^{99 \mathrm{~m}} \mathrm{Tc}$-phosphate compounds, J Nucl Med 16: 1109 (1975).

6. Biello DR, Coleman E, and Stanley RJ: Correlation of renal images on bone scan and intravenous pyelogram, Am J Roentgenol 127: 633 (1976).

7. Adams KJ, Shuler SE, Witherspoon LR, and Neely H: A retrospective analysis of renal abnormality detected on bone scans, Clin Nucl Med 5: 1 (1980).

8. Sty JR, Babbit DP, and Kun L: Atlas of ${ }^{99 m} \mathrm{Tc}-$ methylene diphosphonate renal images in pediatric oncology, Clin Nucl Med 4: 122 (1979).

9. Friedland GW: Uroradiology: an integrated approach, New York, Churchill Livingston, 1983. 